\title{
Brain Tumor Detection using Enhanced Convolution Neural Network for MR Images
}

\author{
M.B. Bramarambika, M Sesha Shayee
}

\begin{abstract}
Brain tumor is a mass that grows unevenly in the brain and directly affects human life. The mass occurs spontaneously because of the tissues surrounding the brain or the skull. There are two types of Brain tumor such as Benign and Malignant. Malignant brain tumors contain cancer cells and grow quickly and spread through to other brain and spine regions as well. Accurate and prompt diagnosis of brain tumors is essential for implementing an effective treatment of this disease. Brain images produced by the Magnetic Resonance Imaging (MRI) technique are a rich source of data for brain tumor diagnosis and treatment in the medical field. Due to the existence of a large number of features compared to the other imaging types. The performance of existing methods is inadequate considering the medical significance of the classification problem. Earlier methods relied on manually delineated tumor regions, prior to classification. This prevented them from being fully automated. The automatic algorithms developed using CNN and its variants could not achieve an influential improvement in performance. In order to overcome such an issue, the proposed one is automatic brain tumor detection system, which is " Enhanced Convolution Neural Network (CNN) Algorithm for MRI Images" for the detection of brain tumor is useful to detect and classify the Glioma part into low Glioma and high Glioma.
\end{abstract}

Keywords: MRI, Malignant, Brain Tumor, Classification, Convolution, Network

\section{INTRODUCTION}

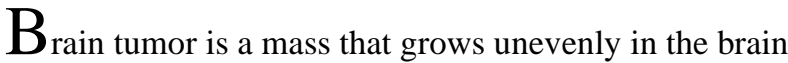
and directly affects human life. The mass occurs spontaneously because of the tissues surrounding the brain or the skull. There are two types of Brain tumor such as Benign and Malignant. Benign brain tumors do not contain cancer cells and grow gradually. They do not spread and commonly stay in one region of the brain, whereas malignant brain tumors contain cancer cells and grow quickly and spread through to other brain and spine regions as well. Malignant or cancerous or a tumor term meaning is a disease in which abnormal growth of cells divide without control and spread to nearby tissues that means other parts of the body through blood and the lymphatic system. Glioma is a type of tumor that starts in the Glial cells of the brain or the spine. Gliomas comprise about 30 percent of all brain tumors and central nervous system tumors, and 80 percent of all malignant brain tumors. Gliomas begin in the gluey supportive cells (Glial cells) that surround nerve cells and help them function.

Manuscript received on June 02, 2021

Revised Manuscript received on June 17, 2021.

Manuscript published on July 30, 2021.

* Correspondence Author

M.B. Bramarambika*, Research Scholar, Gandhi Institute of Technology and Management (GITAM), (Deemed to be University) Vishakhapatnam (A.P), India. Email: mbambika@gmail.com

Dr. M Sesha Shayee, Assistant Professor, Gandhi Institute of Technology and Management (GITAM), (Deemed to be University) Vishakhapatnam (A.P), India. Email: mseshashayee@gmail.com

(C) The Authors. Published by Blue Eyes Intelligence Engineering and Sciences Publication (BEIESP). This is an open access article under the CC BY-NC-ND license (http://creativecommons.org/licenses/by-nc-nd/4.0/)
This undesired and uncontrolled growths of cells is either normally found in the different part of the brain such as Glial cells, neurons, lymphatic tissue, blood vessels, pituitary and pineal gland, skull, or spread cancers mainly located in other organs. Any growth inside such restricted space can cause problem. Brain tumor detection is an important application in medical field, because it provides anatomical information of human abnormal tissues in brain which help the doctors in planning and arranging the treatment.

In hospitals to detect the tumor picture archiving and communication system is used, which can provide either 3D or single slice image. To scan whole 3D or 2D image is time consuming process, hence it is better to use Single Slice image which is associated with the foci within the brain. According to the Pathological Brain Tumor Detection System the Single Slice Imaging is itself the MRI brain imaging System which is also known as Automatic Tumor Detection or Neuro Imaging method. It has two ways to detect the tumor. One is structural imaging and another one is functional imaging The Magnetic Resonance Imaging (MRI) uses magnetic field, not X-rays, to produce detailed images of the body. MRI can be used to measure the tumor size. It scans the brain and creates clearer picture. MRI is the current technology which enables the detection, diagnosis and evaluation.

Accurate and prompt diagnosis of brain tumors is essential for implementing an effective treatment of this disease. . Brain images produced by the Magnetic Resonance Imaging (MRI) technique are a rich source of data for brain tumor diagnosis and treatment due to the existence of a large number of features compared to the other imaging types. Once MRI shows that there is a tumor in the brain, the most common way to determine the type of brain tumor is look at the result of MRI images. In order to overcome such an issue, this paper addresses regarding the automatic detection proposed an Enhanced Convolution Neural Network (CNN) for the detection of brain tumor.

Firstly, we collect the data the means to collect the brain tumor MRI images. We collected these images from kaggle. Next the data augmentation is done used to tackle the data imbalance issue in the data. The augmentation techniques such as cropping to crop the part of the image that contains only the brain, resizing to have every image in similar sizes, normalization to scale pixel values in the range $0-1$ were used. Next the Preprocessing is taken place, which is known filtering the image in order known the edges and regions of the images. the preprocessed images are classified by using the Enhanced CNN classifier. The proposed Enhanced CNN classifies the images as normal or abnormal.

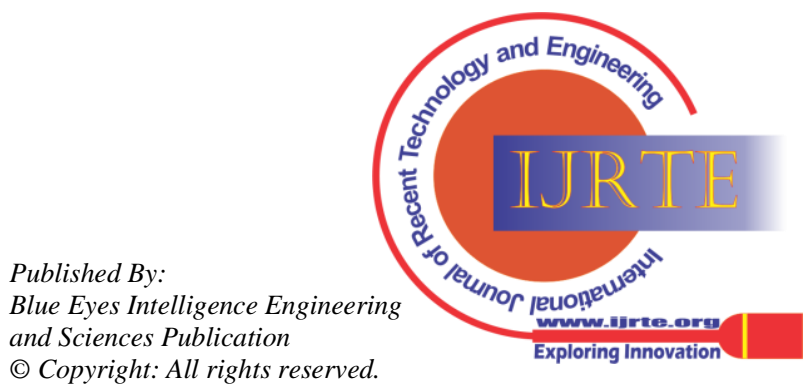




\section{RELATED WORK}

Brain tumor should be detected automatically is somewhat very difficult, because the tumor part cannot be easily detected where the tumor occurs and what status it does have in the situation. In the brain $80 \%$ of tumor can occurs in Glioma parts only. To classify this Glioma into low and high is also somewhat difficult. In recent year we are using spectral based images like single slice and multiple slices are known MRI images which are helpful to our research, they are playing very cautious role in our medical sector. These are all computer aided technologies are giving the doctors very useful theme to find the disease very easily and also there are coming very closer to clinical applications.

Very firstly we collect the images from different sources. And after collecting the image, we use some operations to remove unwanted spectral disturbance from the images using augmentation operation, after the preprocessing operation is going to take place. Then we use the proposed method modalities of images of Enhanced CNN technology with residual batch normalization process to extracting the features and classify and detect the tumor. Before this proposed method we verified many existing methods such as K-Mean, C-Mean Fuzzy C-Mean, and GK fuzzy and so on, but no such method having the accuracy of detecting and classify the Glioma part low and high. The proposed the tumor in right way. Here in the proposed methodology, the Enhanced Convolution Neural Network with Residual Batch Normalization process is used to detect the tumor after verifying number of MRI images in the minute operations in the right way.

\section{PROPOSED METHODOLOGY}

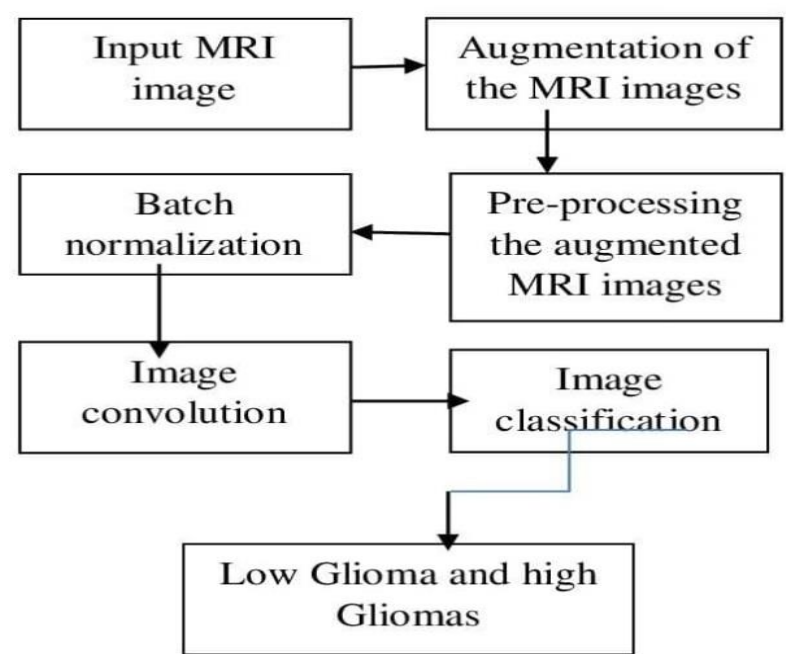

Figure.1: Proposed Methodologies

The proposed is the image classification technique, which is used the tumor oriented images and can do the augmentation, preprocessing, convoluted the images in order safe the image the border and shapes to identify the tumor portion of the MRI images. Images are not easy to directly apply to our process for the classification so we need the different technologies for the purpose. This system better fitting to dataset due to the reduction of number of parameters involved. This proposed one can be trained to understand the sophisticated of the image feature extraction and classification.

\subsection{Methodology}

The brain images are scanned or took from database. They might have some noise. These can be enhanced and contrasted using the augmented techniques; these images can be fed to the preprocessing algorithm, then after fed to the network algorithms to do the extraction and classification. The classification result is Low Glioma and high Glioma. The low Gliomas is no tumor and high Gliomas means there is tumor affected to the patient.

\subsection{Input Images.}

The following is the procedure how to form magnetic (MRI) image. Protons and neutrons of an atom have an angular momentum which is known as a spin. These spins will cancel when the number of subatomic particles in a nucleus is even. Nuclei with odd number will have a resultant spin. This forms the basis of magnetic resonance imaging (MRI), to scan these images the scanner uses powerful magnets to polarize and excite hydrogen nuclear (single proton) in human tissue, which produces a signal that can be detected. MRI Image modality is one of the best image diagnostic techniques when compared to other imaging modalities such as computed Tomography (CT) and X-ray because of its availability at low price with no harmful radiation exposure and its portability.

\subsection{Augmentation}

The augmentation is process to cropping the images to fit the dataset; here scaling reshaping techniques are used the augmented processes is started after taking the images from a particular database.

\subsection{Pre-Processing}

There are many preprocessing techniques. These are used for noise removal, contrast enhancement and illumination equalization. For noise removal we use various filters such as linear filtering and nonlinear filtering. For contrast enhancement and illumination of the images. In the proposed technique, the noise pixels are identified in the first stage. Then edges are detected in the second stage. In the third stage, noise pixels are restored along detected edges. The main objective of filtering is simply to replace each pixel value in an image with the median value of its neighbors, including itself. When median filters are applied to an image, the pixel values which are very different from their neighboring pixels will be eliminated. By eliminating the effect of such odd pixels, the values are assigned to the pixels that are representative of the values of the typical neighboring pixels in the original image.

\subsection{Image Convolution}

The convolution is the process of adding each element of image to its local neighbor's weights by the kernel the purpose of image convolution is to smooth, sharpen, intensify and enhance the medical image. Convolution is performed between signals spanning along tow mutually perpendicular dimensions (if signals are two dimensional in nature) then it will be referred to as 2D convolution.

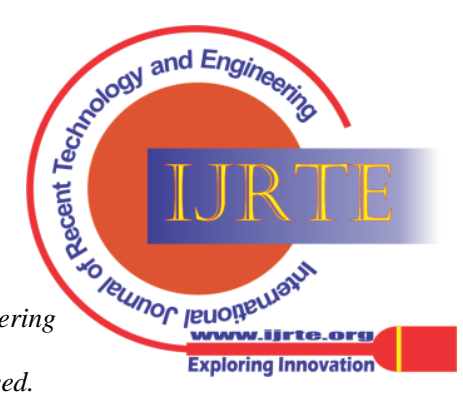


This kind of operation is extensively used in the field of digital image processing where in the $2 \mathrm{D}$ matrix be convoluted with a comparatively smaller matrix called 2D kernel. The image kernel is matrix the features extraction from blur image to non-blur image. In MRI high intensified features of image are extracted in order to classify the image.

\subsection{Batch Normalization}

It is a process to make neural network faster and more stable through adding more layers and fully connected convolution to perform more operation. The batch normalization typically a neural network is trained using a collected set of input segmented images called batch, the normalizing process in these segmented set of images collectively considered to do the operation is known as batch normalization. The normalization is important to the operations.

\subsection{Feature Extraction}

The exraction is process of extraction the Images edges, corner, contours,riges shapes and regions of the MRI Images. The proposed system has mult-layered network in which are many hidden layers to perform this function. In extraction and classification. The convolution layer and max pooling layers perform the feature extraction. The feature exraction is very imporatant because the object can be know perfectectly.

\subsection{Classification}

Full connected consists of the weights and biases along with the neuron and is used to connect the neurons between two different layers. These layers are usually placed before the output layer and form the last few layers of an Enhanced Architecture. The input image from the previous layers are flattened and fed into the FC layer. The flattened vector then undergoes few more FC layers where the mathematical functions operations usually take place. In this stage the classification of the images are taken place. The fully connected layers are more hidden layers. In the neural network it used synaptic weights this synaptic weight due to more connection of neurons in the fully connected layers. In deep learning process the network is trained due to more neurons. Weighted activation function calculates the weight by backward propagation, and then tests the images into Glioma ad non Glioma images.

\section{RESULT ANALYSIS}

\subsection{K-means clustering}

Another one existing technique compared is $\mathrm{K}$ - means segmentation algorithm. The K-mean clustering is hard clustering means no overlapping of cluster is occurred. It is centroids based cluster. A single pixel is compared to all cluster centers using distance formula. The pixel is moved to particular shortest distance cluster among all. Then centroid is re-estimated no of times. Again, each pixel is compared to all centroid. The fundamental drawbacks of $\mathrm{K}$ Mean clustering is K-Mean cluster the number of clusters must be pre-determined proposed system, it does help the system the fastest features. Features are parts or patterns of an object in an image that helps to identify it. There are many features like this proposed system contains the combination of feature

\subsection{Fuzzy C Mean Clustering}

It is a soft clustering technique which is centroid based cluster in which a single data point belongs to more than one cluster. The clusters are overlapped by single data point. . Each pixel of the image is given particular membership value. The fundamental drawbacks of this algorithm are Clustering data of varying sizes and density, Clustering outlier; Scaling with number of dimensions and it is problems of prototypic

\subsection{GK fuzzy Clustering}

The Gustafson Kessel algorithm connects each cluster with both a point and a matrix, respectively representing the cluster center and its covariance. The primary element of this method is its utilization of covariance matrix and distance matrix which makes it possible for clusters to have different shapes. G-K fuzzy utilizes binary classification of brain MR picture as ordinary or tumor influenced. G-K fuzzy partitions the given data into subsets which are gradual. Dimensionality reduction and precise feature set given as input to the G-K fuzzy on the duration of training part as well as during the testing part. The drawback of GK fuzzy algorithm. It requires complex computational time and tumor detection is difficult if more clusters exist

\subsection{Enhanced Based Convolution Neural Network Clustering}

This is the proposed clustering algorithm. It uses many layers. In Enhanced Convolution Neural Network, the Multi-Layered Perception Neural Network structure is used. In this the sigmoid function and activation function output can be calculated. So the network is trained with many images. In the calculation the frequently occurred some dataset are calculated. The gray values of the datasets very nearer, then they are considered as tumor affected portion. In this one there residual system is utilized i.e., some of the stages are skipped if there no more required. In this regard. The activation function sums these weights and gives to the output layer. If the sum of synaptic weights is strong, it decides High Grade Glioma and if the sum of the synaptic weight is weak, it decides Low Grade Glioma by which the tumor affected area is determined

Table 1: Shows the result of different algorithms

\begin{tabular}{|c|c|c|}
\hline Algorithms & $\begin{array}{c}\text { Accuracy } \\
\text { locating part of } \\
\text { the tumor }\end{array}$ & $\begin{array}{c}\text { Accuracy on the } \\
\text { spreading area of } \\
\text { tumor }\end{array}$ \\
\hline K-Mean & 77 & 57 \\
\hline Fuzzy C -Mean & 79 & 68 \\
\hline GK Fuzzy & 83 & 77 \\
\hline Enhanced CNN & 89 & 90 \\
\hline
\end{tabular}

Published By:

Blue Eyes Intelligence Engineering and Sciences Publication

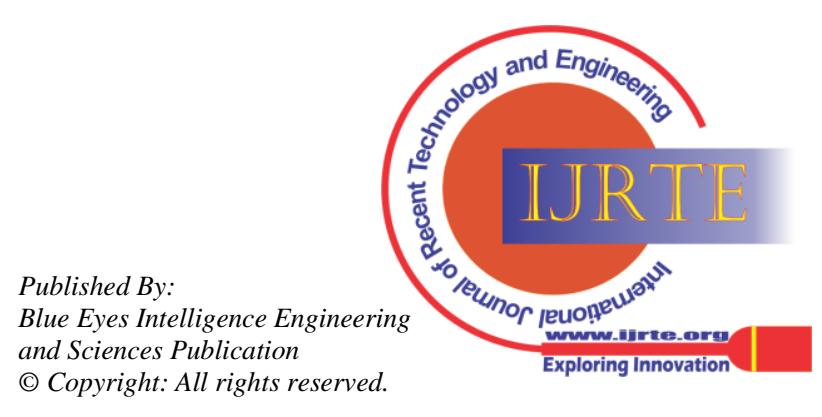




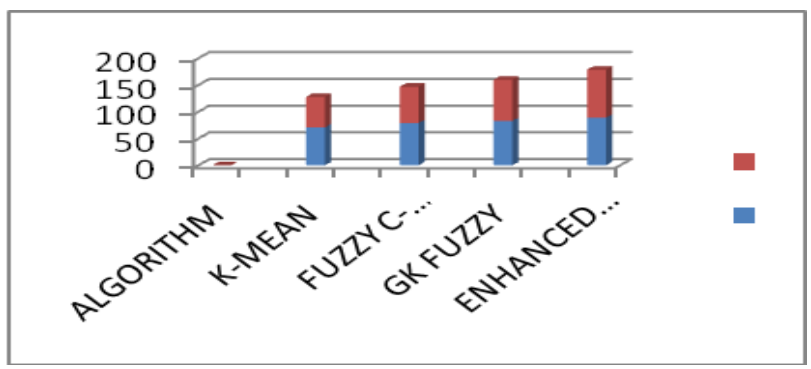

Chart of the results of all algorithms

\section{CONCLUSION}

The traditional approach of detecting MRI based manual inspection, which has become in appropriate for vast volume of data. The computer aided automatic detection tools very important to the neurologist. In the contemporary study this proposed work is verified many techniques on medical image or on magnetic image. It is digital image which is taken by spectra oriented devices, therefore MRI images are known as spectral images. To segment these images is very difficult. In the earlier day no such accuracy method is found in density based algorithms like K-mean, C-mean, fuzzy C-mean and GK Fuzzy. But this proposed work provides the good contrast in feature extraction and classification image comparing prediction to testing images. So we attain the accuracy up to $98 \%$. It is more than enough to detect the tumor in earlier stage.

\section{REFERENCE}

1. Hari Babu Nandpuru, Dr. S. S. Salankar, Prof. V. R. Bora, "MRI Brain Cancer Classification Using Support Vector Machine”, 2014 IEEE Students' Conference on Electrical, Electronics and Computer Science, Year 2014.

2. The IEEE Engineering in Medicine and Biology Society, 2014.

3. Fergana, K., D. Lui, C. Scharffenberger, A. Wong, and D. A. Clausi, "Hybrid Structural and Texture Distinctiveness Vector Field Convolution for Region Segmentation", Computer Vision And Image Understanding Vol. 125, pp. 85 - 96, March, Year 2014.

4. S. Bauer, R. Wiest, L. Nolte, and M. Reyes, "A survey of MRIbased medical image analysis for brain tumor studies," Physics in medicine and biology, vol. 58, no. 13, p. R97, Year 2013.

5. Selvakumar J., Lakshmi A., Arivoli, T., "Brain tumor segmentation and Fuzzy C mean algorithm", International Conference on Advances in Engineering, Science and Management (ICAESM), Year, 2012,

6. B. Vijayakumar, A. Chaturvedi, "Automatic Brain Tumors Segmentation of MR Images using Fluid Vector flow and Support vector Machine", Research Journal of Information Technology, 4(3): 108-114, Year 2012.

7. Amelard, R., A. Wong, and D. A. Clausi, "Extracting Morphological High-Level Intuitive Features (HLIF) for Enhancing Skin Lesion Classification 1] ", 34th Annual International Conference of the IEEE Engineering in Medicine and Biology Society, San Diego, pp. 4458 - 4461, August, Year 2012.

8. S. C. Amartur, D. Piraino, and Y. Takefuji, "Optimization neural networks for the segmentation of magnetic resonance images",IEEE Trans. Med. Imag. , vol. 11, no. 2, pp. no Year 2012

9. Laxmi and P.Samata, "Optimization of visual presentation of MRI image for accurate detection of tumor in human brain using virtual instrument" in The Biomedical Engineering International Conference Year 2012

10. Amelard, R., A. Wong, and D. A. Clausi, "Extracting Morphological High-Level Intuitive Features (HLIF) for Enhancing Skin Lesion Classification 1 ", 34th Annual International Conference of the IEEE Engineering in Medicine and Biology Society, San Diego, pp. 4458 - 4461, August, 2012

11. K.Thapaliya and G.Kwon, "Extraction of brain tumor based on morphological operations," in Proceedings in IEEE-8th international and its area calculation in brain MR images using K-mean clustering

Conference on Computing Technology and Information Management, pp. 515-520, Year 2012

12. T Kalaiselvi and K Somasundaram, "Fuzzy c-means technique with histogram based centroid initialization for brain tissue segmentation in MRI of head scans" in Proceedings in IEEE-international Symposium on Humanities, Science and Engineering Research, pp. 149-154, Year 2011.

13. M.Shasidhar, Y.S.Raja and B.Y.Kumar, "MRI brain image segmentation using modified fuzzy c-means clustering algorithm," in Proceedings in IEEE-International Conference on Communication Systems and Network Technologies, pp. 473-478, Year 2011.

14. S.Koley and A.Majumder, "Brain MRI segmentation for tumor detection using cohesion based self-merging algorithm," in Proceedings in IEEE-3rd International Conference on Communication Software and Networks, pp. 781-785, Year 2011.

15. Kohley S, Majumder A, "Brain MRI segmentation for tumor detection using cohesion based self merging algorithm", IEEE Trans, Current Med. Image. Vol 28, No 4, pp.no.781-785, Year 2011.

16. El-Sayed Ahmed El-Dahshan, Tamer Hosny, Abdel-Badeeh M. Salem "Hybrid intelligent techniques for MRI brain image classification" ELSEVIER Digital Signal Processing, vol. 20, pp433-441, 2010.

17. (2002) The IEEE website. [Online]. Available: http://www.ieee.org/Gopal N.N, Karnan N. M, "Diagnosis of brain tumor through MRI using image processing", IEEE Trans, Current Med. Image. Vol 3, No 35, pp.no.1-4, Year 2010.

18. H. Men, Y. Gao, Y. Wu and X. Li, "Study on Classification Method Based on Support Vector Machine," 2009 First International Workshop on Education Technology and Computer Science, Wuhan, Hubei, pp. 369-373. Year 2009,

19. Kharrat A and Benamrane N, "Detection of brain tumor in medical images", IEEE Trans, Pattern Recog Vol 4, No 39, pp.no.1-6,Year 2009

20. V. Raad, "Design of Gabor wavelets for analysis of texture features in cervical imaging", in Proc. IEEE 25th Annu. Int. Conf., vol. 1, pp. no. 806 - 813, year, 2003.

\section{AUTHORS PROFILE}

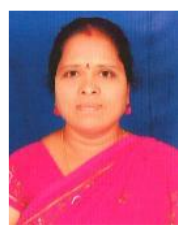

M.B. Bramarambika, has pursuing her $\mathrm{Ph} . \mathrm{D}$ in Computer Science, in the Department of Computer Science, GIS, GITAM (Deemed to be University), Visakhapatnam, Andhra Pradesh. Presently working as Assistant Professor in the Department of Computer Science and Engineering, Telangana University, Telangana State. Her research interests include Image processing and Mobile Networks. She has 12 years of teaching experience. She has published more than 15 publications in International Journals. She has attended 10 conferences and many workshops.

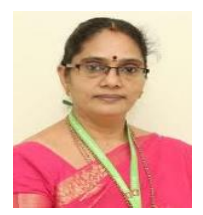

Dr. M.Seshashayee, has been awarded $\mathrm{Ph} . \mathrm{D}$ in Computer Science and Technology, and presently working as Assistant Professor in the Department of Computer Science, GIS, GITAM (Deemed to be University), Visakhapatnam, Andhra Pradesh. Her research interests include Image processing, Internet of things, Big data and Cloud Computing. She has 17 years of teaching experience. She has published more than 25 publications in International Journals. She has attended 20 conferences and many workshops. She is member of CSI and IAENG. is completed MCA M.Tech, and Ph.D. She has 15 years of qualitative teaching experience with taught various subjects like Programming In Java, Internet programming, Software Engineering, Object Oriented Software Engineering, Operating Systems, Software Quality and Testing, Digital Logic Design, Computer Organization and Architecture, Programming in C, Accounting and Financial Management, Embedded Systems, Systems Programming, Information Systems and Organization Behaviour and Her area of research is image segmentation using data mining techniques Image Segmentation Methods using Data Mining Techniques. She has published $11+$ research papers in reputednternational and national journals/ conferences. She attended 20+ conferences, 6 workshops. She is on dynamic part in scholarly and regulatory works in different associations. She is member of CSI and reviewer IJ
Blue Eyes Intelligence Engineering (C) Copyright: All rights reserved. and Sciences Publication

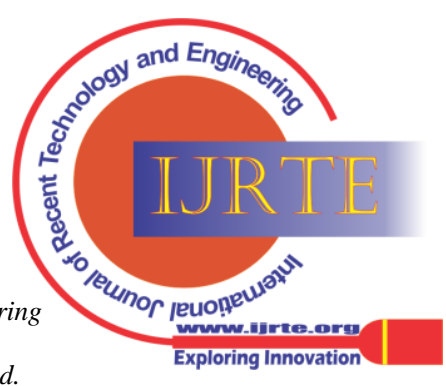

